\title{
ULTRABORNOLOGICAL BOCHNER INTEGRABLE FUNCTION SPACES
}

\section{J.C. Ferrando}

If $(\Omega, \Sigma, \mu)$ is a finite measure space and $X$ is a normed space such that $X^{*}$ has the Radon-Nikodym property with respect to $\mu$, we show first that each space $L_{p}(\mu, x), 1<p<\infty$, is ultrabornological whenever $\mu$ is atomless. When $\mu$ is arbitrary, we prove later on that the space $L_{p}(\mu, X)$ is ultrabornological if $X^{*}$ has the Radon-Nikodym property with respect to $\mu$ and $X$ is itself an ultrabornological space.

In what follows, $(\Omega, \Sigma, \mu)$ will be a finite measure space and $X$ a normed space. If $1 \leqslant p<\infty, L_{p}(\mu, X)$ stands for the space of all (equivalence classes of) $X$-valued Bochner integrable functions $f$ on $\Omega$ with $\int_{\Omega}\|f\|^{p} d \mu<\infty$, provided with the norm

$$
\|f\|=\left(\int_{\Omega}\|f(\omega)\|^{p} d \mu(\omega)\right)^{1 / p}
$$

In this paper a Hausdorff locally convex spaces is said to be ultrabornological if it can be represented as the locally convex hull of all its Banach subspaces with a basis.

If the measure $\mu$ is atomless and the Banach space $X^{*}$ has the Radon-Nikodym property with respect to $\mu$, we are going to show first that $L_{p}(\mu, X)$ is an ultrabornological space for each $1<p<\infty$, regardless of whether or not $X$ is ultrabornological. This must not be a surprising fact if we take into account the two following results concerning finite atomless measure spaces which has been proved recently.

(A): (Drewnowski, Florencio and Paúl, [5], Theorem 3). Let $(\Omega, \Sigma, \mu)$ be a finite measure space and $X$ be a normed space. If the measure $\mu$ is atomless, then $L_{p}(\mu, X)$ with $1 \leqslant p<\infty$ is barrelled.

(B): (Díaz, Florencio and Paúl [2, Main Theorem]). Let $(\Omega, \Sigma, \mu)$ be a finite measure space and $X$ be a normed space. If the measure $\mu$ is atomless, then every weak bounded subset of the dual of $L_{\infty}(\mu, X)$ is bounded in norm; in other words $L_{\infty}(\mu, X)$ is barrelled.

Received 20th January 1992.

Copyright Clearance Centre, Inc. Serial-fee code: 0004-9729/93 \$A2.00+0.00. 
Supposing that $\mu$ is an arbitrary finite measure on $\Omega$ and $X$ is an ultrabornological space such that $X^{*}$ has the Radon-Nikodym property with respect to $\mu$, we shall prove later on that the dense subspace $S_{p}(\mu, X)$ of $L_{p}(\mu, X)$, with $1<p<\infty$, of all countably valued functions, is ultrabornological. In the particular case when $\mu$ is purely atomic, this implies that each normed space $\ell_{p}\{X\}, 1<p<\infty$, of absolutely $\ell_{p}$-summable sequences $[6, p .139]$ with the usual norm, is ultrabornological. Since each finite measure space decomposes into a purely atomic part and an atomless part, the previous results imply that if $\mu$ is an arbitrary finite measure on $\Omega, X$ is ultrabornological and $X^{*}$ has the Radon-Nikokym property with respect to $\mu$, then the space $L_{p}(\mu, X), 1<p<\infty$, is ultrabornological.

THEOREM 1. Let $1<p<\infty$. If the measure $\mu$ is atomless and $X^{*}$ has the Radon-Nikodym property with respect to $\mu$, then $L_{p}(\mu, X)$ is ultrabornological.

Proof: Suppose there exists some $p, 1<p<\infty$ such that $L_{p}(\mu, X)$ is not an ultrabornological space. Then, there is an absolutely convex set $V$ in $L_{p}(\mu, X)$ that meets each Banach subspace $F$ with a basis of $L_{p}(\mu, X)$ in a neighbourhood of the origin in $F$, but $V$ is not itself a neighbourhood of the origin in $L_{p}(\mu, X)$. We are going to begin the proof building inductively a sequence $\left\{\left(\Omega_{n}, \Sigma_{n}, \mu_{n}\right), \quad n=1,2, \ldots\right\}$ of atomless finite measure spaces satisfying for each $n \in \mathbb{N}$ the following properties

(i) $\Omega_{n} \in \Sigma_{n-1}, \Omega_{n+1} \subseteq \Omega_{n}, \mu\left(\Omega_{n}\right)=\mu\left(\Omega_{n-1}\right) / 2$

(ii) $\Sigma_{n}=\Sigma_{n-1 \mid \Omega_{n}}, \mu_{n}=\mu_{n-1 \mid \Sigma_{n}}$

(iii) $V$ does not absorb the unit sphere of $L_{p}(\mu, X)$.

The atomless character of $\mu$ assures the existence of an $A \in \Sigma$ such that $\mu(A)=\mu(\Omega) / 2$. By restricting $\Sigma$ and $\mu$ to $A$ and its complement $\Omega \backslash A$ we obtain the atomless measure spaces $(A, \mathcal{A}, \lambda)$ and $\left(\Omega \backslash A, \mathcal{A}^{\prime}, \lambda^{\prime}\right)$ so that $L_{p}(\mu, X)$ is the topological direct sum of $L_{p}(\lambda, X)$ and $L_{p}\left(\lambda^{\prime}, X\right)$. If $W$ and $W^{\prime}$ denote the unit spheres of $L_{p}(\lambda, X)$ and $L_{p}\left(\lambda^{\prime}, X\right)$, respectively, then it is clear that $V$ either does not absorb $W$ or it does not absorb $W^{\prime}$. Thus, setting $\left(\Omega_{1}, \Sigma_{1}, \mu_{1}\right)$ to be either $(A, \mathcal{A}, \lambda)$, or $\left(\Omega \backslash A, \mathcal{A}^{\prime}, \lambda^{\prime}\right)$, depending on whether $V$ does not absorb $W$ or does not absort $W^{\prime}$, we have accomplished the first step of our induction process.

Now assuming $\left(\Omega_{i}, \Sigma_{i}, \mu_{i}\right), 1 \leqslant i \leqslant n$, are already defined, we proceed to build $\left(\Omega_{n+1}, \Sigma_{n+1}, \mu_{n+1}\right)$ with the former requirements.

The atomless character of $\mu_{n}$ yields a certain $A_{n} \in \Sigma_{n}$ such that $\mu_{n}\left(A_{n}\right)=$ $\mu_{n}\left(\Omega_{n}\right) / 2$. As before, $L_{p}\left(\mu_{n}, X\right)$ can be descomposed as the topological direct sum of $L_{p}\left(\lambda_{n}, X\right)$ and $L_{p}\left(\lambda_{n}^{\prime}, X\right)$, where $\left(A_{n}, \mathcal{A}_{n}, \lambda_{n}\right)$ and $\left(\Omega_{n} \backslash A_{n}, \mathcal{A}_{n}^{\prime}, \lambda_{n}^{\prime}\right)$ are the respective restrictions of $\Sigma_{n}$ and $\mu_{n}$ to $A_{n}$ and $\Omega_{n} \backslash A_{n}$. Since $V$ does not absorb the unit sphere $V_{n}$ of $L_{p}\left(\mu_{n}, X\right)$, it cannot absorb both $W_{n}$ and $W_{n}^{\prime}$ (the unit spheres of $L_{p}\left(\lambda_{n}, X\right)$ and $L_{p}\left(\lambda_{n}^{\prime}, X\right)$, respectively). Hence, we choose again $\left(\Omega_{n+1}, \Sigma_{n+1}, \mu_{n+1}\right)$ to be either $\left(A_{n}, \mathcal{A}_{n}, \lambda_{n}\right)$ or $\left(\Omega_{n} \backslash A_{n}, \mathcal{A}_{n}^{\prime}, \lambda_{n}^{\prime}\right)$, according to whether $V$ does not 
absorb $W_{n}$ or does not absorb $W_{n}^{\prime}$. This ends our induction process.

For each $n \in \mathbb{N}$ we select an element $h_{n} \in V_{n}$ such that $h_{n} \notin n V$, and then we form the sequence $\left(h_{n}\right)$ contained in the unit sphere of $L_{p}(\mu, X)$, with the support of each $h_{n}$ contained in $\Omega_{n}$.

Define $E:=\bigcap_{k=1}^{\infty} \Omega_{k}$ and note that $\mu(E)=\lim _{n \rightarrow \infty} \mu\left(\Omega_{n}\right)=0$.

Since $X^{*}$ has the Radon-Nikodym property with respect to $\mu$, then $L_{q}\left(\mu, X^{*}\right)$ with $1 / p+1 / q=1$ is the topological dual of $L_{p}(\mu, X)$, (see [3, p.98]) and we are going to see next that $\left\langle f, h_{n}\right\rangle \rightarrow 0$ for each $f \in L_{q}\left(\mu, X^{*}\right)$.

In fact, if $p>1$ and $f \in L_{q}\left(\mu, X^{*}\right)$, then

$$
\begin{aligned}
\left|\left\langle f, h_{n}\right\rangle\right|^{q} & =\left|\int_{\Omega}\left\langle f(\omega), h_{n}(\omega)\right\rangle d \mu(\omega)\right|^{q}=\left|\int_{\Omega_{n}}\left\langle f(\omega), h_{n}(\omega)\right\rangle d \mu(\omega)\right|^{q} \\
& =\left|\left\langle f e\left(\Omega_{n}\right), h_{n}\right\rangle\right|^{q} \leqslant\left\|f e\left(\Omega_{n}\right)\right\|^{q}=\int_{\Omega_{n}}\|f(\omega)\|^{q} d \mu(\omega) .
\end{aligned}
$$

But, as $\omega \rightarrow\|f(\omega)\|^{q} \in L_{1}(\mu)$ and $\mu\left(\Omega_{n}\right) \rightarrow 0$, it follows that

$$
\lim _{n \rightarrow \infty} \int_{\Omega_{n}}\|f(\omega)\|^{q} d \mu(\omega)=0
$$

and, therefore, $\left\langle f, h_{n}\right\rangle \rightarrow 0$.

This shows that $\left(h_{n}\right)$ is a weakly null normalised sequence in $L_{p}(\mu, X)$. Consequently, the Bessaga-Pelczynski selection principle, [3], guarantees the existence of a subsequence $\left(h_{n_{i}}\right)$ of $\left(h_{n}\right)$ which is a basic sequence in $L_{p}(\mu, \widehat{X})$.

Define finally $g_{j}(\omega)=h_{j}(\omega)$ if $\omega \notin E$ and $g_{j}(\omega)=0$ otherwise. So $g_{j}$ is equivalent to $h_{j}$ for each $j \in \mathbb{N}$ and on the one hand we have

$$
g_{n} \notin n V
$$

for each $n \in \mathbb{N}$ while, on the other hand, $\left(g_{n_{i}}\right)$ is a basic sequence in $L_{p}(\mu, X)$. Now, since supp $g_{n_{i}} \subseteq \Omega_{n_{i}} \backslash E, \Omega_{n_{i+1}} \subseteq \Omega_{n_{i}}$ for each $i \in \mathbb{N}$ and $\cap\left\{\Omega_{n_{i}} \backslash E, i \in \mathbb{N}\right\}=\emptyset$, it is easy to notice that every element $g$ of the closed linear cover $G$ of the sequence $\left(g_{n_{i}}\right)$ in $L_{p}(\mu, \widehat{X})$ belongs to $L_{p}(\mu, X)$. Indeed, $g(\omega) \in X$ for each $\omega \in \Omega$. Hence, $G$ is a Banach subspace of $L_{p}(\mu, X)$.

Given that $V$ meets $G$ in a neighbourhood of the origin in $G$, this leads to the existence of some positive integer $q$ such that

$$
g_{n_{q}} \in n_{q} V .
$$

This contradiction ends our proof. 
REMARK. If we apply the previous argument to the dense subspace $S_{p}(\mu, X)$ of $L_{p}(\mu, X)$ formed by all $X$-valued functions with countably many values, it also shows that this subspace is ultrabornological whenever $\mu$ is atomless and $X^{*}$ has the RadonNikodym property with respect to $\mu$.

In the sequel $(\Omega, \Sigma, \mu)$ will be an arbitrary finite measure space. If $M$ is a set of a linear space $L,\langle M\rangle$ stands for the linear hull of $M$ in $L$. By $S(\mu, X)$ we shall denote the space of all $X$-valued $\mu$-simple functions defined on $\Omega$. On the other hand, given $1<p<\infty$ and $E \in \Sigma$, the spaces $S\left(\left.\mu\right|_{E}, X\right)$ and $S_{p}\left(\left.\mu\right|_{E}, X\right)$ will be denoted simply by $S(E, X)$ and $S_{p}(E, X)$, respectively.

Given $f \in S_{p}(\mu, X)$, suppose that $g$ is some appropriate canonical representing of $f$ having only countably many values. If $x \in \Im g$ then by $[1$, p.167] we have that $g^{-1}(x) \in \Sigma$. Hence, $\left\{g^{-1}(x), x \in \Im g\right\}$ is a countable partition of $\Omega$ formed by pairwise non-empty elements of $\Sigma$ such that $f$ is essentially constant in each of its members.

Lemma 1. Let $\left\{A_{n}, n \in \mathbb{N}\right\}$ be a pairwise disjoint sequence of non-empty elements of $\Sigma$. Assume that $X^{*}$ has the Radon-Nikodym property with respect to $\mu$. If $V$ is an absolutely convex set in $S_{p}(\mu, X)$ which meets each Banach subspace $F$ with a basis in a neighbourhood of the origin in $F$, then there exists an $m \in \mathbb{N}$ such that $V$ absorbs the closed unit ball of $S_{p}\left(\cup\left\{A_{n}, n>m\right\}, X\right)$.

Proof: If the property is not true, $V$, does not absorb the closed unit ball of $S_{p}\left(\cup\left\{A_{n}, n>m\right\}, X\right)$ for each $m \in \mathbb{N}$. Hence, for each $m \in \mathbb{N}$ there is some $f_{m} \in$ $S_{p}\left(\cup\left\{A_{n}, n>m\right\}, X\right)$ such that $\left\|f_{n}\right\|=1$ and

$$
f_{m} \notin m V .
$$

If we set $\Omega_{m}:=\cup\left\{A_{n}, n>m\right\}$, then $\left(f_{m}\right)$ is a normalised sequence in $S_{p}(\mu, X)$ such that $\operatorname{supp} f_{m} \subseteq \Omega_{m}$ for each $m \in \mathbb{N}$. Since $X^{*}$ has the Radon-Nikodym property with respect to $\mu$ and $\mu\left(\Omega_{m}\right) \rightarrow 0$, then we may proceed as in Theorem 1 in order to show that $\left\langle h, f_{n}\right\rangle \rightarrow 0$ for every $h \in L_{q}\left(\mu, X^{*}\right)$ with $1 / p+1 / q=1$. Therefore, $\left(f_{n}\right)$ is a weakly null normailised sequence in $L_{p}(\mu, X)$ and the Bessaga-Pelczynski selection principle establishes the existence of a subsequence $\left(f_{n_{i}}\right)$ of $\left(f_{n}\right)$ which is a basic sequence in $L_{p}(\mu, \widehat{X})$. Now, as $\Omega_{n+1} \subseteq \Omega_{n}$ for each $n \in \mathbb{N}$ and $\cap\left\{\Omega_{n}, n \in \mathbb{N}\right\}=\emptyset$, it is easy to notice that every element $f$ of the closed linear span $F$ of the sequence $\left(f_{n_{i}}\right)$ in $L_{p}(\mu, \widehat{X})$ is $X$-valued and has countably many values. Consequently, $F$ is a Banach subspace of $S_{p}(\mu, X)$.

Given that $V$ meets $F$ in a neighbourhood of the origin in $F$, the Baire category theorem leads to the existence of some positive integer $q$ such that

$$
f_{n_{q}} \in n_{q} V .
$$


This contradiction ends our proof.

Lemma 2. Let $V$ be an absolutely convex set in $S_{c}(\mu, X)$ meeting each Banach subspace $F$ with a basis of $S_{p}(\mu, X)$ in a neighbourhood of the origin in $F$. If $X$ is ultrabornological and $X^{*}$ has the Radon-Nikodym property with respect to $\mu$, then $V$ absorb the closed unit ball of $S(\mu, X)$.

Proof: Suppose the property is not true. Since $V$ does not absorb the unit sphere of $S(\mu, X)$, there is some $f_{1} \in S(\mu, X)$ with $\left\|f_{1}\right\|=1$ such that

\section{$f_{1} \notin 2 V$.}

Let $\left\{Q_{11}, Q_{12}, \ldots, Q_{1 k(1)}\right\}$ be a partition of $\Omega$ by non-empty sets of $\Sigma$ such that $f_{1}$ takes a different constant value $\mu$-almost everywhere in each $Q_{11}$ with $1 \leqslant i \leqslant k(1)$. Now, as $S(\mu, X)$ is the topological direct sum of the subspaces $S\left(Q_{11}, X\right), 1 \leqslant i \leqslant$ $k(1)$, there is some $m(1) \in\{1,2, \ldots, k(1)\}$ such that $V$ does not absorb the unit sphere of $S\left(Q_{1 m(1)}, X\right)$. Thus, there is some $f_{2} \in S\left(Q_{1 m(1)}, X\right)$ with $\left\|f_{2}\right\|=1$ such that

$$
f_{2} \notin 4 V \text {. }
$$

Again, there exists a finite partition $\left\{Q_{21}, Q_{22}, \ldots, Q_{2 k(2)}\right\}$ of $Q_{1 m(1)}$ by nonempty sets of $\Sigma$ such that $f_{2}$ is constant $\mu$-almost everywhere in each set $Q_{2 i}, 1 \leqslant$ $i \leqslant k(2)$ and takes a different value. As $S\left(Q_{1 m(1)}, X\right)$ is the topological direct sum of the subspaces $S\left(Q_{2 i}, X\right), 1 \leqslant i \leqslant k(2)$ and $V$ does not absorb the unit sphere of $S\left(Q_{1 m(1)}, X\right)$, there is some $m(2) \in\{1,2, \ldots, k(2)\}$ such that $V$ does not absorb the unit sphere of $S\left(Q_{2 m(2)}, X\right)$. Hence, there is some $f_{3} \in S\left(Q_{2 m(2)}, X\right)$ with $\left\|f_{3}\right\|=1$ such that

$$
f_{3} \notin 6 T \text {. }
$$

Proceeding by recurrence we obtain a sequence $\left(f_{n}\right)$ of $\mu$-simple functions and a sequence $\left(\Omega_{n}\right)$, with $\Omega_{n}=Q_{n, m(n)}$ for each $n \in \mathbb{N}$, of sets in $\Sigma$, verifying that, for each $n \in \mathbb{N}$,

(i) $\left\|f_{n}\right\|=1$

(ii) $\operatorname{supp} f_{n+1} \subseteq \Omega_{n}$

(iii) $f_{n}$ is essentially constant in $\Omega_{n}$

(iv) $\Omega_{n+1} \subseteq \Omega_{n}$

(v) $f_{n} \notin 2 n V$

Let $P:=\bigcap_{i=1}^{\infty} \Omega_{i}$. Two cases are in order depending on whether $\mu(P)$ is or is not different from zero.

If $\mu(P) \neq 0$ and for each $n \in \mathbb{N} x_{n}$ denotes the constant value of $f_{n} \mu$-almost everywhere in $\Omega_{n}$, we define $h_{j}(\omega)=f_{j}(\omega)$ if $\omega \notin P$ and $h_{j}(\omega)=x_{j}$ if $\omega \in P$ for 
each $j \in \mathbb{N}$. Then we write

$$
h_{j}^{\prime}:=h_{j}-x_{j} e(P)
$$

for all $j \in \mathbb{N}$.

Since $x \rightarrow e(P) x$ is an isometry from $X$ into $S_{p}(\mu, X)$, the ultrabornology of $X$ guarantees that the set $V \cap e(P) X$ is a neighbourhood of the origin in $e(P) X$, which leads to the existence of some $r \in \mathbb{N}$ such that $x_{i} e(P) \in r V$ for each $i \in \mathbb{N}$. Hence,

$$
x_{n} e(P) \in n V
$$

for each $n \geqslant r$, consequently,

$$
h_{j}^{\prime} \notin j V
$$

for each $j \geqslant r$. Finally, we define $g_{j}=h_{j}^{\prime} /\left\|h_{j}^{\prime}\right\|$ for each $j \in \mathbb{N}$. Since $\left\|h_{j}^{\prime}\right\| \leqslant 1$ for each $j \in \mathbb{N}$, it follows that

$$
g_{j} \notin j V
$$

for each $j \geqslant r$. Clearly $\left(g_{n}\right)$ is a normalised sequence with supp $g_{n} \subseteq \Omega_{n} \backslash P$ for each $n \in \mathbb{N}$ and $\cap\left\{\Omega_{n} \backslash P, n \in \mathbb{N}\right\}=\emptyset$.

If $\mu(P)=0$, for each $j \in \mathbb{N}$, we take $g_{j}(\omega)=f_{j}(\omega)$ if $\omega \notin P$ and $g_{j}(\omega)=0$ otherwise, for each $j \in \mathbb{N}$. Then $g_{j}$ is equivalent to $f_{j}$ for each $j \in \mathbb{N}$ and consequently,

$$
g_{j} \notin j V
$$

for each $j \in \mathbb{N}$. As $\left\|g_{j}\right\|=\left\|f_{j}\right\|=1$ for each $j,\left(g_{n}\right)$ is normalised. On the other hand, we have again that $\operatorname{supp} g_{j} \subseteq \Omega_{j} \backslash P$ for each $j \in \mathbb{N}$.

Since $\mu\left(\operatorname{supp} g_{n}\right) \rightarrow 0$ in both cases, we proceed as in the previous results to show that $\left(g_{n}\right)$ is weakly null in $L_{p}(\mu, X)$ and consequently that it contains a basic sequence $\left(g_{n_{i}}\right)$ in $L_{p}(\mu, \widehat{X})$. As supp $g_{n} \subseteq \Omega_{n} \backslash P$ for each $n \in \mathbb{N}$ and $\cap\left\{\Omega_{n} \backslash P, n \in \mathbb{N}\right\}=\emptyset$, it is easy to show that the closed linear span $\left[g_{n_{i}}\right]$ in $L_{p}(\mu, \widehat{X})$ is contained in $S_{p}(\mu, X)$. This yields a contradiction.

Theorem 2. Let $1<p<\infty$ and suppose that $X$ is ultrabornological. If $X^{*}$ has the Radon-Nikodym property with respect to $\mu$, then $S_{p}(\mu, X)$ is ultrabornological.

Proof: Assume that $X$ is ultrabornological but $S_{p}(\mu, X)$ is not. Then there is some $f_{1} \in S_{p}(\mu, X)$ with $\left\|f_{1}\right\|=1$ such that

$$
f_{1} \notin 2 V \text {. }
$$

As we noticed before, there is a partition $\left\{Q_{1 i}, i \in \mathbb{N}\right\}$ of $\Omega$ by non-empty sets of $\Sigma$ such that $f_{1}$ is essentially constant in each set $Q_{1 i}$. By Lemma 1 there is an $n_{1} \in \mathbb{N}$ 
such that $V$ does absorb the closed unit ball of $S_{p}\left(U\left\{Q_{1 n}, n>n_{1}\right\}, X\right)$. So $V$ does not absorb the unit sphere of $S_{p}\left(\cup\left\{Q_{1 n}, n \leqslant n_{1}\right\}, X\right)$.

Let $\Omega_{1}:=\cup\left\{Q_{1 n}, n \leqslant n_{1}\right\}$. Since $V$ does not absorb the unit sphere of $S_{p}\left(\Omega_{1}, X\right)$, there is some $f_{2} \in S_{p}\left(\Omega_{1}, X\right)$ with $\left\|f_{2}\right\|=1$ such that

$$
f_{2} \notin 3 V \text {. }
$$

Let $\left\{Q_{2 i}, i \in \mathbb{N}\right\}$ be a partition of $\Omega_{1}$ formed by non-empty sets of $\Sigma$ such that $f_{2}$ is essentially constant in each $Q_{2 i}$. Applying Lemma 1 again, there is an $n_{2} \in \mathbb{N}$ such that $V$ does absorb the closed unit ball of the subspace $S_{p}\left(\cup\left\{Q_{2 n}, n \geqslant n_{2}\right\}, X\right)$. Hence, defining $\Omega_{2}:=\cup\left\{Q_{2 i}, i \leqslant n_{2}\right\}, V$ cannot absorb the unit sphere of $S_{p}\left(\Omega_{2}, X\right)$ and thus there is some $f_{3} \in S_{p}\left(\Omega_{2}, X\right)$ with $\left\|f_{3}\right\|=1$ such that

$$
f_{3} \notin 4 V \text {. }
$$

Proceeding by recurrence we obtain a sequence $\left(f_{n}\right)$ of functions of $S_{p}(\Omega, X)$ and a sequence $\left(\Omega_{n}\right)$ of sets in $\Sigma$ verifying for each $n \in \mathbb{N}$ the following properties
(i) $\left\|f_{n}\right\|=1$
(ii) $\operatorname{supp} f_{n+1} \subseteq \Omega_{n}$
(iii) $e\left(\Omega_{n}\right) f_{n} \in S\left(\Omega_{n}, X\right)$
(iv) $\Omega_{n+1} \subseteq \Omega_{n}$
(v) $f_{n} \notin(n+1) V$.

For each $j \in \mathbb{N}$ we set

$$
g_{j}:=f_{j}-e\left(\Omega_{j}\right) f_{j}
$$

Clearly, $e\left(\Omega_{j}\right) f_{j} \in S(\mu, X)$ for each $j \in \mathbb{N}$ and taking into account Lemma 2, there is no loss of generality in assuming that

$$
e\left(\Omega_{j}\right) f_{j} \in V
$$

for each $j \in \mathbb{N}$. This implies that

$$
g_{j} \notin j V
$$

for each $j \in \mathbb{N}$.

It is clear that supp $g_{i} \cap \operatorname{supp} g_{j}=\emptyset$ if $i \neq j$, and it is not difficult to see from this fact that the closed linear span $\left[g_{j}\right]$ in $L_{p}(\mu, \widehat{X})$ of the sequence $\left(g_{j}\right)$ is a copy of $\ell_{p}$ which is contained in $S_{p}(\mu, X)$. Now the Baire category theorem leads to the existence of some $k \in \mathbf{N}$ such that

$$
g_{k} \in k V \text {. }
$$

This contradiction ends the proof. 
Corollary. Suppose that $X$ is ultrabornological and $\mu$ is a purely atomic, measure on $\Omega$. Then $L_{p}(\mu, X)$ is ultrabornological.

ProOF: If $\mu$ is purely atomic $X^{*}$ has the Radon-Nikodym property with respect to $\mu$ and $L_{p}(\mu, X)$ is isometric to $\ell_{p}\{X\}$. So, the conclusion follows from Theorem 2.

TheOREM 4. Let $(\Omega, \Sigma, \mu)$ be an arbitrary finite measure space and let $X$ be an ultrabornological normed space. If $X^{*}$ has the Radon-Nikodym property with respect to $\mu$, then $L_{p}(\mu, X)$ with $1<p<\infty$ is ultrabornological.

Proof: Since each finite measure space containing some atom decomposes into a purely atomic part and an atomless part, this is an obvious consequence of Theorem 1 and the previous Corollary.

\section{REFERENCES}

[1] B. Beauzamy, Introduction to Banach spaces and their geometry, Mathematics Studies 68 (North Holland, 1982).

[2] J.C. Díaz, M. Florencio and P.J. Paúl, 'A uniform boundedness theorem for $L_{\infty}(\mu, E)$ ', Arch. Math. (to appear).

[3] J. Diestel, Sequences and series in Banach spaces, Graduate Texts in Math. 92 (Springer-Verlag, Berlin, Heidelberg, New York, 1984).

[4] J. Diestel and J.J. Uhl Jr., Vector Measures, Mathematical Surveys No. 15. (A.M.S., Providence, Rhode Island, 1977).

[5] L. Drewnowski, M. Florencio and P.J. Paúl, 'The space of Pettis integrable functions is barrelled', Proc. Amer. Math. Soc. 114 (1992), 687-694.

[6] P. Pérez Carreras and J. Bonet, Barrelled locally convex spaces, Mathematics Studies 131 (North Holland, 1987).

E.U. Informática

Depto. Matematica Aplicada

Universidad Politécnica de Valencia

Apartado 22012, E-46071 Valencia

Spain 